\title{
Epidemiological models of SARS-CoV-2 (COVID-19) to control the transmission based on current evidence: A systematic review
}

\author{
Swati Arora ${ }^{1}$, Rishabh Jain ${ }^{1}$, Harendra Pal Singh 1,* \\ ${ }^{1}$ Cluster Innovation Centre, University of Delhi, Delhi-110007, India
}

\begin{abstract}
In Wuhan city of China, an episode of novel coronavirus (COVID-19) happened. during late December and it has quickly spread to all places in the world. Until May 29, 2020, cases were high in the USA with 1.7 Million, Russia with approximately 387 thousand, the UK with 271 thousand confirmed cases. Everybody on the planet is anxious to know when the coronavirus pandemic will end. In this scourge, most nations force extreme medication measures to contain the spread of COVID-19. Modeling has been utilized broadly by every national government and the World Health Organization in choosing the best procedures to seek after in relieving the impacts of COVID-19. Many epidemiological models are studied to understand the spread of the illness and its prediction to find maximum capacity for human-to-human transmission so that control techniques can be adopted. Also, arrangements for the medical facilities required such as hospital beds and medical supplies can be made in advance. Many models are used to anticipate the results keeping in view the present scenario. There is an urgent need to study the various models and their impacts. In this study, we present a systematic literature review on epidemiological models for the outbreak of novel coronavirus in India. The epidemiological models with environmental parameters such as water, temperature, humidity, and air on the
\end{abstract}

\footnotetext{
*Corresponding author E-mail address: harendramaths@gmail.com (Harendra Pal Singh)
} 
dynamics of COVID-19 is also studied. Here, In addition, an attempt to take out the results from the exploration and comparing it with the real data. The study helps to choose the models that are progressive and dependable to predict and give legitimate methods for various strategies.

Keywords: SARS-COV-2, COVID-19, Lockdown, Epidemiological Models, Machine Learning, Transmission.

\section{Introduction}

Coronavirus is very infectious and can induce disorder in individuals with multiple complexities. It can lead to Middle-East-Respiratory-Syndrome (MERS) and Severe-Acute-Respiratory-Syndrome (SARS). It has now tainted practically all pieces of the world as yet unfurling. The introductory cases were recorded in December 2019 and presumably spread by the contact through your eye, nose, and mouth. Signs differ from individual to individual after infection leading to fever, throbbing-painfulness, nasal-blockage, migraine, conjunctivitis, sore-throat, loose-bowels, loss-of-taste, and smell, or a rash on skin or staining of fingers or toes. These side-effects are typically gentle and start consistently while some become contaminated and take a serious adverse effect on health [1]. The episode of the SARS happened in 2003 which had originated the earlier year in Asia and arbitrary cases in different parts on the planet were found, the World-Health-Organization (WHO) stated that a novel-coronavirus recognized by various research centers was the causative agent for SARS. The infection was officially named the SARS-coronavirus (SARS-CoV). Over 8 thousand individuals were sullied, around $10 \%$ of whom passed on. In September 2012, another form of coronavirus was found, initially called novel-coronavirus-2012, and now officially named 
Middle-East-Respiratory- Syndrome-coronavirus (MERS-CoV) was found. About 2500 cases of MERS-CoV illness were affirmed by the laboratory and approximate 850 died by December 2019.

Wuhan encountered an episode of outbreak of coronavirus that killed over eighteen hundred and tainted more than seventy thousand people within the initial fifty days of the spread by the end of 2019. The International committee on Taxonomy of viruses (ICTV) named the infection as SARS-CoV-2 and the ailment as COVID-19. This infection was supposed to be a member of the group of coronaviruses. The infection was also named as Wuhan-coronavirus or 2019-Novel-coronavirus by the Chinese authorities [2].

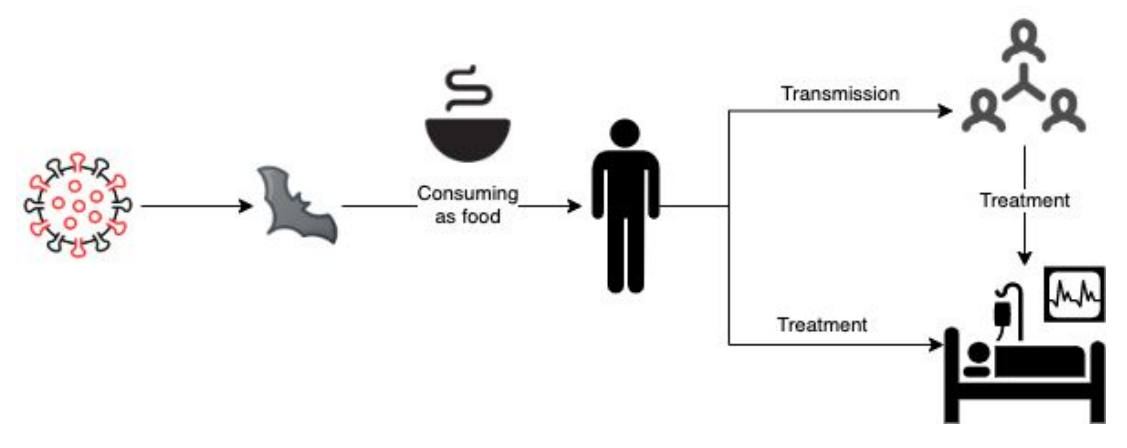

Fig. 1: Showing the flow diagram of Origin of COVID-19 (Betacoronavirus)

In the beginning, the number of cases multiplied roughly every-week. On 20 January, China revealed about 150 new cases in a day. Later official information shows more than 6000 people had just developed manifestations by that point, and more may have been contaminated. On 30 January, WHO pronounced the coronavirus a crisis of worldwide concern. WHO thought about Europe as the dynamic focal point of the pandemic starting on 13 March. Retesting of earlier cases found an individual in France who had the infection on 27 December 2019 and an individual in the United States who passed on of the illness on 6 February $[3,4]$. Iran turned into 
a focal point of the spread of the infection after China during February. The outbreak may have been more serious than the around 400 cases detailed by the Iranian government by that date as demonstrated by more than ten nations following their cases back to Iran by 28 February. The Iranian government revealed a hundred deaths in a single day, the most recorded in the nation since the outbreak started on 15 March. By 23 March, Iran was encountering fifty new cases each hour and new passing like clockwork due to coronavirus.

COVID-19 was affirmed to have reached South Korea on 20 January. The country's wellbeing office announced a noteworthy increment in affirmed cases on 20 February. On 28 February, over 2,000 affirmed cases were accounted for, ascending to 3,150 on 29 February. WHO has considered Europe the dynamic focal point of the pandemic starting on 13 March. Cases by a nation over Europe had multiplied multiple times in commonly 3 days, with certain nations (for the most part those at prior phases of identification) indicating multiplying like clockwork [5]. On 31 January, The outbreak was confirmed to have spread to Italy. The Council of Ministers reported another announcement law to contain the outbreak, including isolating over 50,000 individuals from eleven distinct regions in northern Italy on 22 February. Italy overwhelmed China as the nation with the most coronavirus related deaths on the planet in the wake of detailing around 3400 fatalities from the pandemic on 19 March. Official figures uncovered that Britain had the most exceedingly terrible COVID-19 loss of life in Europe, provoking an investigation into the treatment of the pandemic on 5 May. The loss of life in the United Kingdom was about 30 thousand in COVID patients.

The initial cases were accounted for in the United States in January. U.S. turned into the nation with the most elevated number of affirmed COVID-19 patients on 26 March, with more than 82 thousand cases. And with a high mortality rate of 20 thousand 11 April. The death rate 
due to COVID-19 was 1.5 million with around 100 thousand deaths on 15 May 2020. On 4 May, Canada announced 60 thousand cases and around 4000 deaths, while Mexico revealed 23 thousand cases and 2000 deaths. On 11 March, WHO perceived the spread of COVID-19 as a pandemic. Also Eurasia detailed flooding cases.

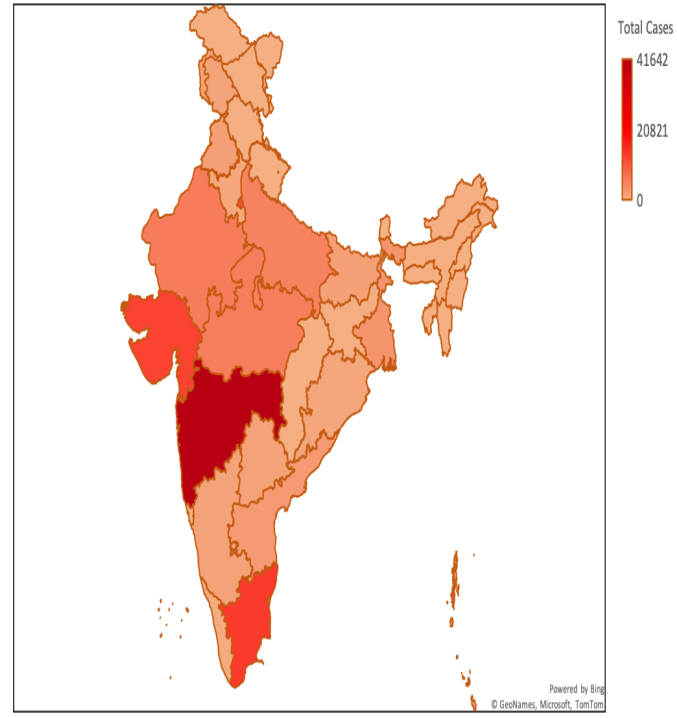

(a)

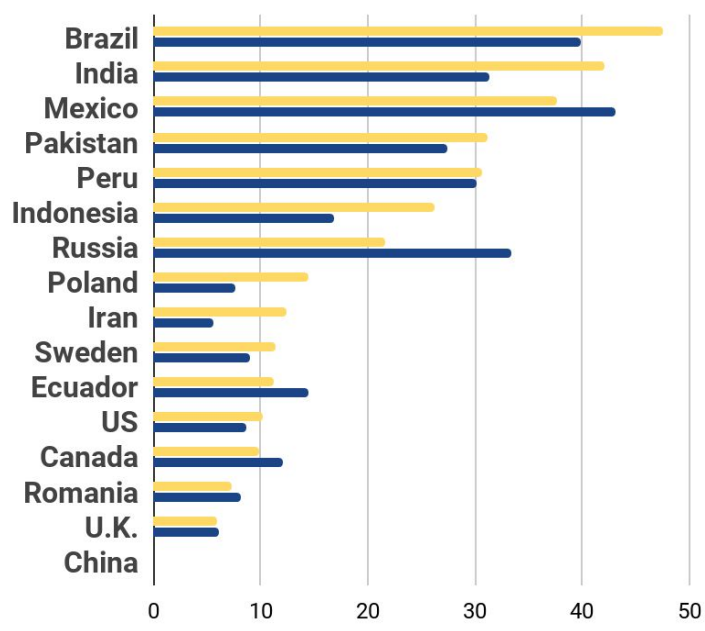

Change in active cases over the past seven days (in \%)

Change in deaths over the past seven days (in \%)

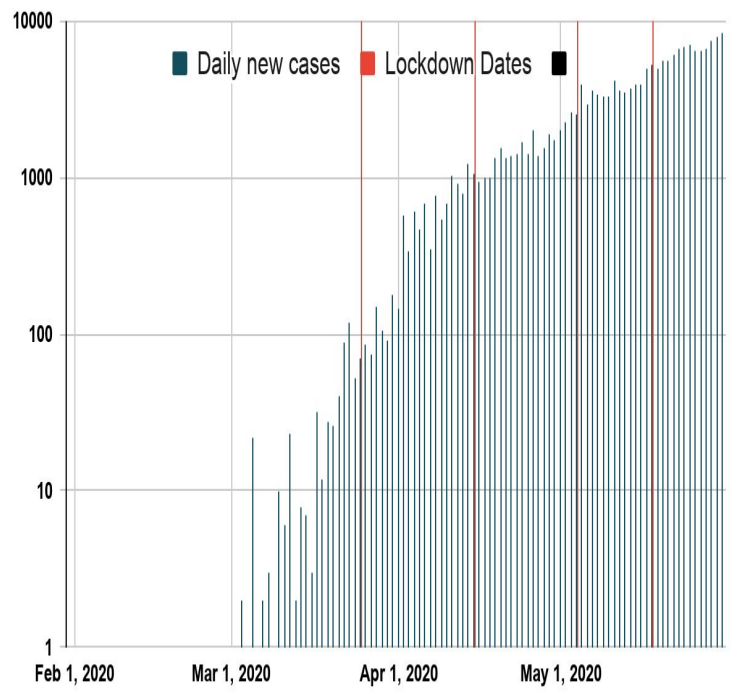

(b)

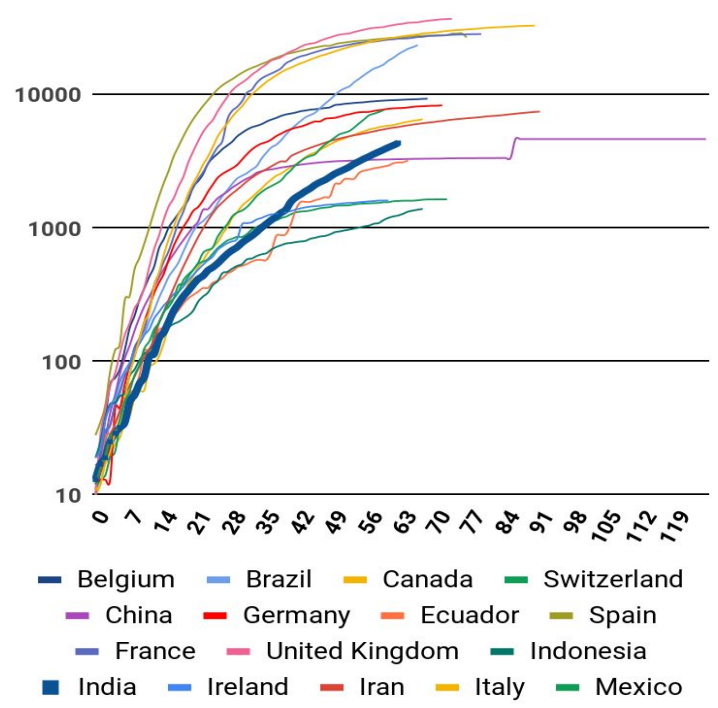


(c)

(d)

Fig. 2 : (a) Map of India showing state Wise total cases, (b) Variation in daily new confirmed cases (c) Variation of active cases and deaths in various countries, (d) Showing the number of cases increase since the $100^{\text {th }}$ cases

In India on 30 January, the first case was a Kerala's Thrissur locale who got back from Wuhan University in China. On 2 February, the subsequent case was reported in Kerala's Alappuzha locale who also had come back from Wuhan, China. On 3 February, the third case was accounted for in Kerala's Kasaragod region consequently. On 3 March, the spouse of the Italian visitor in Jaipur likewise tested positive, and her samples were sent to Pune for affirmation. An aggregate of 24 individuals (21 Italians and 3 Indians) dwelling in a South Delhi lodging were moved to an ITBP camp for testing. The list proceeded with most of them having headed out history to different nations. On 12 March first affirmed deaths due to COVID were recorded in India. Till 15 March, India has its initial 100 confirmed cases. 22 March, one day Janta Curfew was imposed and passenger air travel suspended. 25 March, Nationwide lock-down forced till 14 April. Lockdown reached out in various stages and began giving some contingent consents from its third stage (Shown in Figure 2b).

India had its initial 1000 cases and 100 recouped on 30 March. 31 March, an enormous bunch was recognized under the Delhi district [6]. 14 April, India recorded 10,000 affirmed cases which multiplied by 22 April and hit 30,000 tallies by 29 April additionally arriving at 1000 deaths. Affirmed cases arrived at 50,000 on 7 May and arrived at 100,000 on 19 May. On the bright-side, the recovered check likewise arrived at 20,000 on 11 May. From the above (Figure 2a), we can clearly see that the cases are highest in Urban Areas. This may be due to the high density in the regions. Also, the main reason might be due to the high number of testing done in 
the urban area. The recovery rate is also increasing, reaching about $90 \%$ in various cities, and reaching about $50 \%$ in major cities like Delhi. According to the above map, most cases can be seen in the west most to the central parts of India. This map shows the number of cases is highest in states which includes the major industrial cities of the country. Figure 2c shows the changes in active cases, as well as deaths. From Figure $2 \mathrm{~d}$, we can conclude that the cases since the 100th case are high in India as compared to other countries [7].

Epidemiological models planned for understanding the human-to-human transmission and the control techniques to stop the transmission. The mathematical modeling studies may help to provide evidence and insights into the transmission through the environmental medium like air, and water. For India, there are different models used to decipher the information and predict the results of COVID-19. There are epidemiological models displaying the investigation of COVID-19 including the models like dynamic models, machine learning, and statistical models. Considering these examinations we are assessing the ends by recognizing qualities, confinements, and potential usage. In this paper, we are trying to take out the outcomes from the research and comparing the outcomes with the real-life scenario and evaluating their strengths, limitations, and potential implementations. This paper is divided into three sections as the introduction, mathematical models of COVID-19, and conclusion/discussions.

\section{Epidemiological models of COVID-19}

Mathematical models are being extensively used in the study of disease expansion and forecasting worldwide. Epidemiological-models are quite effective for foretelling the number of fresh cases or for identifying the best measures to diminish transmission. The researchers throughout the world have studied numerous mathematical-modeling and numerical-analysis on COVID-19 since its outbreak. This section will describe the past studies towards the growth of 
various mathematical-models of COVID-19 in India. Critical features of these models are a lifelike portrayal of the long-term physiological behavior, forecast of COVID-19 cases, and its prevention. Some of these forecasts also include environmental factors that are affected due to conditions like temperature, humidity, and transmittance through water and air.

\subsection{SIR Models}

Practically all numerical models of sicknesses start from a similar fundamental reason: that the populace can be partitioned into a lot of particular classes, subordinate upon their involvement regarding the infection. Easiest of these models order people as susceptible, infectious, or recovered. This is named the SIR model. People are naturally introduced to the susceptible class. Susceptible people have never come into contact with the malady and can come down with the ailment, after which they move into the infectious class. Infectious people spread the malady to susceptible and stay in the infectious class for a given timeframe (the infectious period) before moving into the recovered class. At long last, people in the recovered class are thought to be safe forever. The basic mathematical equations and diagrammatic representation are as follows

$$
\begin{gathered}
d S / d t=-\beta S I / N \\
d I / d t=\beta S I / N-\gamma I \\
d R / d t=\gamma I
\end{gathered}
$$

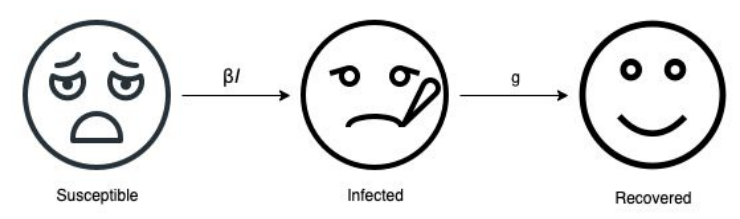

Fig. 3: SIR model diagrammatic representation 
The transmissible-rate, $\beta$, regulates the flow of spread which describes the possibility of spreading infection within a susceptible and infectious-individual. Recovery-degree, $y=1 / D$, is managed by the average span, D, of infection. And $\mathrm{N}$ is the Total-population. Singh R. et al. [8] proposed the SIR model on $25^{\text {th }}$ March 2020 and predicted that a three-week lockdown is insufficient and suggested protocols of sustained lockdown with periodic relaxation. Assuming all cases to be symptomatic so $\alpha=1$. The fit parameter $\beta=0.0155$ and set $\gamma=1 / 7$ with the basic reproductive ratio $R_{0}=2.10$. They suggested a 49-day-long lockdown to eliminate the cases. Compared to real-data after 60-days there is still exponential growth instead of elimination of cases. This might be due to various factors including a high population, less awareness, and insufficient corporation from the people. Or the fact that there was a very less amount of people being tested. Dhar A. [9] presented a modified model including asymptomatic infectious $\left(I^{\mathrm{a}}\right)$ and symptomatic infectious $\left(\mathrm{I}^{\mathrm{s}}\right)$. The proposed model is as follows

$$
\begin{gathered}
d S / d t=-\beta u(t) I / N \\
d I^{a} / d t=\alpha \beta u(t) I S / N-\gamma I^{a} \\
d I^{S} / d t=(1-\alpha) \beta u(t) I S / N+\gamma I^{S} \\
d R / d t=\gamma\left(I^{S}+I^{a}\right)
\end{gathered}
$$

where time-dependent function $u(t)$ is the "lockdown" function that incorporates the effect of a lockdown on the rate of spreading of the infection. They observed that the infected population must be less than 200 by the 60 days of lockdown. But in a few days, their model started contradicting their own results. So, they mentioned that the model fails, even at a qualitative level, right from the time of the lockdown. Ranjan R. [10] used the fit parameter $\beta=0.043, y=$ 0.285 and $R_{0}=1.540$. They predicted the final epidemic size around 13,000 which happens 
around the end of May 2020. But even before the last week of May, we have surpassed the 100,000 counts. This may be due to the incompetence of the USA lockdown data (which he used to calculate parameters) with India's data due to the large difference in population density. Bhola J. et al. [11] used two sets of values $\beta=2$, and $\mathrm{V}=0.01$ in their model and $R_{0}=2.6$. Jhakar M. et al. [12] and Malhotra B. et al. [13] presented the simulation results using fminsearch and ode45 functions of MATLAB provided by Batista, M. [14,15]. According to the prediction of the model till now the total infected cases are very close to the real value but the per day new cases deviate less than $50 \%$ of real value.

Tiwari A. [16] used a modified SIR model (SIQR) with a Quarantined (Q) population. The model equations are represented as

$$
\begin{gathered}
d S / d t=-\beta S I / N \\
d I / d t=\beta S I / N-(\alpha+\eta) I \\
d Q / d t=\eta I-\gamma Q \\
d R / d t=\gamma Q+\alpha I
\end{gathered}
$$

here $\eta$ determines the rate at which new cases are detected and $\alpha$ is the rate of removal of infectious individuals. $\alpha=0.287, \beta=0.476, \gamma=0.040, \eta=0.020, l=0.99942, N=1.3 \times 10^{9}$ and $\mathrm{R}_{0}=1.55$. The resulting prediction values on comparing with real data, the predictions are very low almost $1 / 10$ th of the real values. Yadav R. S. [17] used the SIR model with $\beta / N=0.03156$ and $\gamma=0.0714$. Until now their results fit the real data with very little error. Therefore, their prediction says that the numbers will be maximum on May 25 and then will decrease eventually ending by the first week of August. Kumar S. [18] used the parameters $R_{0}=2.66, \beta=0.5$, 
$\gamma=0.1, N=7000$ and $15000, S_{0}=7000$ and $15000, I_{0}=1$ for the prediction of Maharashtra state in India. Their predicted values are different from real observations. They predict less than 1500 cases by the last week of May but current data has more than 30,000 active cases. Singh A. et.al. [19] used the SIR model with $N=100,000, \beta=0.14013338$ and $\gamma=0.0180899$. Comparing their predictions with the real data none of their described predictions matches real data and there is a difference of at least $20 \%$ from real data. Kumar K. et al. [20] considered the effect of the complete lockdown of the country on the spread of the infection. Their predictions seem to match the current data.

Maji C. [21] used the SIR model with additional factor $E(t)$, where $E(t)$ is the mass of infectious material in the environment. In this model, direct and indirect disease transmission rates are also considered. They predicted that the infected population will end by $170-180$ days from March. Pujari B. S. et al. [22] used a composite approach that practices the SIR model for different cities which are in turn linked via practical transportation-networks that help movement among them. Mandal M. et al. [23] used the SIR model on April 24. In this model, the data-driven measures of basic reproduction number $\left(R_{0}\right)=1.03$, average reproduction number $(R)=1.73$, and effective reproduction number $\left(R_{e}\right)=1.35$, respectively. The results of the exponential and SIR models have shown higher estimates of $R_{0}, R$, and $R_{e}$. In conclusion, they predicted the epidemic size of India was estimated to be around 30 thousand. They predicted the end of the epidemic on June 9. But, current data doesn't show any decrease in numbers. Dhanwant J. N. et al. [24] used the SIR model on May 3. According to their results, the model matches real data to lockdown implemented values from the model. The author doesn't provide any predictions. Das S. [25] used the SIR model to measure the basic reproduction number $\mathrm{R}_{0}$ for India and its states. The confirmed cases for India should stay below 66,224 by May 1 which matches with real data. Deo, V. et.al. [26] used the SIR on May 14. They used a system based 
on the time-series SIR model to determine time-dependent conditioners for the transmission degree of the disease. These conditioners are used in the state-space SIR model. Their predictions are matched with the real data. Furthermore, the model predicted a final epidemic size of 50 million by October end and then decreased in the epidemic size.

\subsection{SIS Models}

The SIS model is utilized for a given final population susceptible to a distinct disease and carries the disease in the area. Ghosh P. et al. [27] used the following SIS model on major states of India - Maharashtra, Tamil Nadu, Delhi, M.P., etc. on April 15, he used the four $\beta$ values individually for each state and calculated the infection rates out of it compared to real data.

$$
\begin{gathered}
d S / d t=-\beta S I / N+\gamma I \\
d I / d t=\beta S I / N-\gamma I
\end{gathered}
$$

\subsection{SIRS and SIRD Models}

In SIRS, the individual becomes susceptible again as resistance wanes, i.e. recovered individuals maintain only short-term resistance or no resistance. And as per the data shown by WHO and MoHFW, there are many cases of the recovered patient being infected again by COVID-19. Hence, this must be considered as one of the major models related to COVID-19. The mathematical equations are given below. The Parameter $\boldsymbol{\xi}$ denotes the degree of recovered individuals returns to the susceptible state

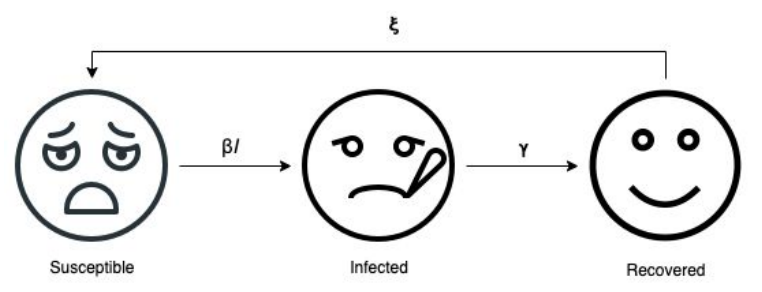




$$
\begin{gathered}
d S / d t=-\beta S I / N+\xi R \\
d I / d t=\beta S I / N-\gamma I \\
d R / d t=\gamma I-\xi R
\end{gathered}
$$

Fig. 4: SIRS model diagrammatic illustration

The Susceptible-Infectious-Recovered-Deceased model distinguishes among recovered (meaning especially people survived the disease and now immune) and deceased. COVID-19 being a deadly disease has a large number of people dying due to it. This model has the following system of differential equations. The new parameter $\boldsymbol{\mu}$ represents the rate of mortality.

$$
\begin{gathered}
d S / d t=-\beta S I / N \\
d I / d t=\beta S I / N-\gamma I-\mu I \\
d R / d t=\gamma I \\
d D / d t=\mu I
\end{gathered}
$$

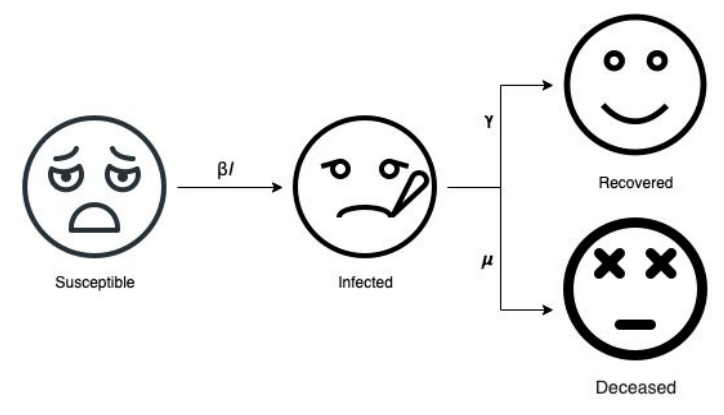

Fig. 5: SIRD model diagrammatic illustration

Rajesh A. et al. [28] used the SIRD model with two parameters set one for optimistic and another for pessimistic scenarios as $\mu=0.135, \beta=0.03, \gamma=0.0035$ and $\mu=0.22, \beta=0.01$, $\gamma=$ 0.0035 , respectively. Considering the current data the pessimistic model resembles it very much. According to this model, the peak will appear after approximately 100 days from March 22, 2020, i.e., around the last week of June 2020. And will reach the infected cases to $10^{9}$. 
Prasad J. [29] proposed a model to fit a set of SIR and SIRD models, with a time-dependent contact rate, to Covid-19 data for a set of 45 most affected countries. They have discussed a set of parametric models for one of the transmission coefficients $\beta(t)$. Till now, the data is insufficient to evaluate which model is accurate. Kumar A. et al. [30] used SIRD on April 17. They have used the Sequential Bayesian method (SBM) for simulation in the SIRD model. In this study, the real infected cases are $10 \%$ of predicted data. Hence, the model fails to predict correctly. Chatterjee S. et al. [31] used the SIRD model with $y=1 / 12, N \sim 10^{6}, \beta=0.257, \boldsymbol{\mu}=$ 0.0032. The predicted values are in range but have a delay of 1 month by now.

\subsection{SEIR Models}

For many diseases, there is a vital incubation phase through which somebody has been affected but is not yet contagious. During this phase, the individual is in Exposed (E) condition. This model is important as many precautions are being taken to prevent exposure of infected persons like Lockdown, curfew, and home quarantining of people. The parameter $\boldsymbol{\alpha}$ is the rate of being infected if exposed. This basic model has the following system of differential equations

$$
\begin{gathered}
d S / d t=\mu N-\mu S-\beta S I / N \\
d E / d t=\beta S I / N-(\mu+\alpha) I \\
d I / d t=\alpha E-(\gamma+\mu) I \\
d R / d t=\gamma I-\mu R
\end{gathered}
$$

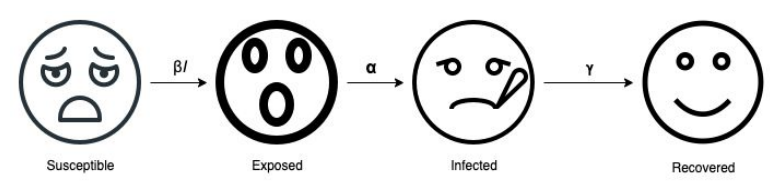

Fig. 6: SEIR model diagrammatic illustration 
Ranjan R. [32] used a modified version of SEIR as SEIQRDP instead of the previously used SIR model. The equations are as follows

$$
\begin{gathered}
d S / d t=-\beta S I / N-\alpha S \\
d E / d t=\beta S I / N-\gamma E \\
d I / d t=\gamma E-\delta I \\
d Q / d t=\delta I-\lambda(t) Q-\kappa(t) Q \\
d R / d t=\lambda(t) Q \\
d D / d t=\kappa(t) Q \\
d P / d t=\alpha S
\end{gathered}
$$

with $Q$ (for quarantined cases - confirmed and infected) and mortality rate $\mathrm{k}(\mathrm{t})=\mathrm{k} 0 \exp (-\mathrm{k} 1 \mathrm{t})$ are time-dependent and average quarantine time $\delta^{-1}$. Predicting estimated total deaths by the end of the epidemic could be around 33000 , which is around $4 \%$ of total estimated cases. For India, predicted value till May 19 is to reach 30,000 . This model also failed, as on 19 May the numbers were even more than 100,000. Maji A. et al. [33] used the same model on May 9 to predict the state-wise predictions on the major states of India. In comparison with real data, the predictions have a difference of around $25 \%$ of the real data. Sardar T. et al. [34] proposed two different models to be used with and without lockdown on April 7. Both models are based on SEIR with added asymptomatic $\mathrm{A}(\mathrm{t})$, hospitalized or notified $\mathrm{C}(\mathrm{t})$ population, and population in the lockdown compartment $L(t)$ - SLEAICR. The model equations with lockdown are as follows

$$
d S / d t=\mu N-\mu S-\beta_{1} S I /(N-L)-\rho \beta_{1} A S I /(N-L)+\omega L-{ }_{1} S
$$




$$
\begin{gathered}
d L / d t=\imath-(\mu-\omega) L \\
d E / d t=\beta_{1} S I /(N-L)+\rho \beta_{1} A S I /(N-L)-(\mu+\alpha) E \\
d A / d t=(1-\kappa) \sigma E-\left(\gamma_{1}+\tau_{1}+\mu\right) A \\
d I / d t=\kappa \alpha E-\left(\gamma_{2}+\tau_{2}+\mu\right) I \\
d C / d t=\tau_{1} A+\tau_{2} I-\left(\delta+\gamma_{3}+\mu\right) C \\
d R / d t=\gamma_{1} A+\gamma_{2} I+\gamma_{3} C-\mu R
\end{gathered}
$$

where $\gamma_{1}$ is recovery rate for asymptomatic infected, $\gamma_{2}$ is the recovery rate for symptomatic infected, $\gamma_{3}$ represents the recovery rate for hospitalized or notified individuals, $\rho$ is the reduction in COVID-19 transmission for asymptomatic infected, $\kappa$ is a fraction of exposed population that become symptomatically infected, $1 / \sigma$ is incubation period for COVID-19, $\delta$ is the death rate of hospitalized or notified population, and $1,1 / \omega$ are lockdown success rate and lock-down period respectively. In this model, the reproduction number $R_{0}$ is defined as

$$
R_{0}=\beta_{1} \kappa \sigma /\left((\mu+\sigma)\left(\gamma_{2}+\tau_{2}+\mu\right)\right)+\rho \beta_{1}(1-\kappa) \sigma /\left((\mu+\sigma)\left(\gamma_{1}+\tau_{1}+\mu\right)\right)
$$

The above model was implemented in three different states and overall India. According to the final results, they predicted that on May 7 the daily cases will go down to zero in Delhi and Tamil Nadu and will reach a peak of 1300 in Maharashtra and around 8000 in overall India. Considering the current data, we had around 450, 580, 1200, and 3344 confirmed cases added on May 7. We found a close relation in the Maharashtra data but there is a huge difference in other states' data. 
Senapati, A. et al. [35] proposed a SEIHR model on April 10. This model includes symptomatic $(\mathrm{I})$, asymptomatic $\left(\mathrm{I}_{\mathrm{a}}\right)$, quarantined $\left(\mathrm{I}_{\mathrm{q}}\right)$, and hospitalized $(\mathrm{H})$ effects. The model is as follows

$$
\begin{gathered}
d S / d t=-\beta S\left(I+\eta I_{a}\right) / N \\
d E / d t=\beta S\left(I+\eta I_{a}\right) / N-\sigma E \\
d I / d t=\rho_{1} \sigma E-\alpha I \\
d I_{a} / d t=\rho_{2} \sigma E-\gamma_{a} I_{a} \\
d I_{q} / d t=\rho_{3} \sigma E-\left(\alpha_{q}+\gamma_{q}\right) I_{q} \\
d H / d t=\alpha I+\alpha_{q} I_{q}-(\gamma+\delta) H \\
d R / d t=\gamma_{a} I_{a}+\gamma_{q} I_{q}+\gamma H
\end{gathered}
$$

They considered $\beta=1.1164, \sigma=0.1923$ as the rate of transition from exposed to infected class, $\eta=0.2$ as modification parameter, $\rho_{1}, \rho_{2}, \rho_{3}=0.5210,0.2740,0.2050$, respectively Fractions of the population move from the compartment $E$ to the compartments I, la and Iq, respectively. They selected $\alpha=0.2174$ as the rate of transition from symptomatic to hospitalized class, $\alpha_{q}=0.1429$ as the rate of transition from quarantined to hospitalized class, $\gamma=0.0701$ as the recovery rate of the individuals in the hospitalized compartment, $Y_{a}=0.13978$ as the recovery rate of the asymptomatic population, $\mathrm{V}_{\mathrm{q}}=0.11624$ as the recovery rate of quarantined population and $\delta=0.0175$ as the rate of disease-induced death. The estimated value of the basic reproduction number $R=4.1849$ is obtained. They found the upper and lower bounds of 
R0 are 4.5014 and 3.8799, respectively. Pal D. et al. [36] proposed a model based on SEIR on April 29. The SEQIR model with a quarantined (Q) population is given as

$$
\begin{gathered}
d S / d t=\Lambda-\alpha S E-\beta S_{1}-\sigma_{1} S-d_{1} S \\
d E / d t=\alpha S E-r_{1} E-\beta_{2} E-d_{1} E \\
d Q / d t=\beta_{1} S+\beta_{2} E-r_{2} Q-\sigma_{2} Q-d_{1} Q \\
d I / d t=r_{1} E+r_{2} Q-\sigma_{3} I-d_{1} I-d_{2} I \\
d R / d t=\sigma_{1} S+\sigma_{2} Q+\sigma_{3} I-d_{1} R
\end{gathered}
$$

They selected $\Lambda=40,000, \beta_{1}=4 \times 10^{7}, \beta_{2}=0.1, \sigma_{1}=5 \times 10^{-4}, \sigma_{2}=5 \times 10^{-2}, \sigma_{3}=6 \times 10^{-3}$, $r_{1}=0.01, r_{2}=0.001, d_{2}=197 \times 10^{-5}$ and $d_{1}=2 \times 10^{-5}$. We found that the infected population reached a max of around $3.8 \times 10^{8}$ on 120 days from March 21. In 60 days it will reach 300,000 which is three times the real value.

Menon A. et al. [37] proposed a modified SEIR model (SEIQCRD) on April 30. This model includes symptomatic $(\mathrm{I})$, asymptomatic $\left(\mathrm{I}_{\mathrm{a}}\right)$, quarantined $(\mathrm{Q})$, hospitalized $\left(\mathrm{Q}^{\prime}\right)$, carrier $(\mathrm{C})$, recovered without disability $\left(R_{w d}\right)$, deceased $(D)$, recovered with disability $\left(R_{d}\right)$. The proposed model has the following form

$$
\begin{gathered}
d S / d t=\alpha(t) S\left(I+I^{\prime}+C\right) / N+g R_{w d} \\
d E / d t=\alpha(t) S\left(I+I^{\prime}+C\right) / N+\mu E \\
d I_{s}^{\prime} / d t=r(1-s) \mu E-h(t) I_{s}^{\prime}
\end{gathered}
$$




$$
\begin{gathered}
d I / d t=r s \mu E-\varepsilon(t) I+f C-\varsigma_{1} I-\eta_{1} I+h(t) I_{s}^{\prime} \\
d I^{\prime} / d t=(1-r) \mu E-\beta_{3} I^{\prime}-\varsigma_{3} I^{\prime}-\eta_{3} I^{\prime}-\lambda(t) I^{\prime} \\
d Q / d t=\varepsilon(t) I-\beta_{1} Q-v Q-\rho Q-\varsigma_{2} Q-\eta_{2} Q+\lambda(t) I^{\prime} \\
d Q^{\prime} / d t=\rho Q-\beta_{4} Q^{\prime}-\varsigma_{5} Q^{\prime}-\eta_{5} Q^{\prime} \\
d C / d t=v Q-f C-\beta_{2} C-\varsigma_{4} C-\eta_{4} C \\
d R_{w d} / d t=\beta_{1} Q+\beta_{2} I^{\prime}+\beta_{2} C-g R_{w d} \\
d D / d t=\varsigma_{1} I+\varsigma_{2} Q+\varsigma_{3} I^{\prime}+\varsigma_{4} C+\varsigma_{5} Q^{\prime} \\
d R_{w d} / d t=\eta_{1} I+\eta_{2} Q+\eta_{3} I^{\prime}+\eta_{4} C+\eta_{5} Q^{\prime}
\end{gathered}
$$

where

$$
\begin{gathered}
\alpha(t)=\alpha 1 H((\tau l-t r)-t)+\left(\left(\left((t-(\tau l-t r))^{p}+(\alpha 1-\alpha 2)\right)\right) \times e^{-(t-(\tau l-t r)) / \tau r}+\alpha 2\right) H(t-(\tau l-t r) \\
\varepsilon(t)=1-0.5 e^{-t / \tau m} \\
h(t)=(1 / \pi) \arctan (d(t-\tau)+0.5 \\
\left.\left.\lambda(t)=0.7\left(1-e^{-t / \tau q}\right) H(t-t) w\right) q\right)
\end{gathered}
$$

The parameter values for simulation results are $N=1,400,000,000, \alpha_{1}=0.5, \alpha_{2}=0.4, s=0.5$, $d=0.5, \beta_{1}=0.5, \beta_{2}=0.1458, \beta_{3}=0.1458, \beta_{4}=0.05, \mu=0.5, v=0.05, \varepsilon=0.5, r=0.3, g=$ $0.0001, f=0.2, \rho=0.01, \tau q=62, \tau I=58, p=0.53, \mathrm{Tw}=55, \varsigma_{1}=0.25, \varsigma_{2}=0.2, \varsigma_{3}=0.1$, $\varsigma_{4}=0.05, \varsigma_{5}=0.2, \eta_{1}=0.1, \eta_{2}=0.1, \eta_{3}=0.05, \eta_{4}=0.01, \eta_{5}=0.1, \tau m=100, \tau q=5000, \operatorname{tr}=$ $5, \tau r=6$. In this model, they evaluated the predictions until April 23 and found that the numbers 
of infected individuals are quite close to the real data until that day. They didn't share any short term prediction in numbers for the country. Only they predicted that if lockdown is lifted even after 100 days i.e. July 6 , their model predicted 1,460,000 deaths and 4,133,000 infected beings in India. Singh A. et al. [38] used the SEIR model with the deceased (D). The results of their model for both optimistic and pessimists are failing to resemble current data, the pessimist result has a $30 \%$ increased result from current data. Sinha D. N. [39] proposed a SEAIQIm model on May 4. They used asymptomatic infectious $(A)$, quarantine $(Q)$, recovered humans with Immunity (Im). The model equations are given as

$$
\begin{gathered}
d S / d t=B N-\beta i S I-\beta S A-\beta S Q-\mu S \\
d E / d t=\beta i S I+\beta A S A+\beta e S Q-(\mu+\eta+\xi+\sigma) E \\
d A / d t=\xi E-(\lambda+\theta+\mu) A \\
d I / d t=\eta E-(\mu+\gamma) I \\
d Q / d t=\gamma I+\lambda A-(\mu+\delta+\alpha) Q \\
d I m / d t=\alpha Q+\theta A+\sigma E-\mu \operatorname{Im} \\
N(t)=S(t)+E(t)+A(t)+I(t)+\operatorname{Im}(t) \\
d N / d t=B-\mu N-\delta Q \leq B-\mu N
\end{gathered}
$$

India reached its peak reproduction number on March $23\left(R_{0}=34.9\right)$. Khatua D. et al. [40] proposed a model based on SEIR on May 10. The model includes asymptomatically infected $(A)$, symptomatically infected $(I)$ as SEIAR. They utilized a dynamic modeling way to illuminate the estimations of factors and afterward predicted numerous consequences of various factors 
and relied upon the control conditions. Mandal S. et al. [41] used two reproduction numbers $\mathrm{R}_{0}=$ 1.5 and 4 for optimistic and pessimist, respectively. They also used the SEIQR model for four major states in India. They proposed multiple conclusions including optimistic/pessimist for with/without interventions. Pai C. et al. [42] used the SEIR model on April 28. The parameter $\beta$ is optimized during the fitting procedure. The results predicted that the infected cases will be at a max of 43,000 in mid-May but current data shows cases crossing 100,000 around mid-May. Hence, the prediction failed this may be due to the fact that the ratio in which people the interventions are different or the population density is very different in both countries. Chowdhury R. et al. [43] used SEIPHURD with infected individuals $(P)$, the hospitals $(H)$, and patients who stay in ICU (U). The proposed model is given as

$$
\begin{gathered}
d S / d t=-I S \\
d E / d t=I S-E \\
d I / d t=E-I \\
d P / d t=f I-P \\
d H / d t=P-H \\
d U / d t=f U H-U \\
d R / d t=(1-f) I+(1-f) H+(1-f) U \\
d D / d t=f D U
\end{gathered}
$$

The parameters values are selected as $1 / \omega=2 \cdot 7,1 / \sigma=5 \cdot 2,1 / \gamma=2 \cdot 3,1 / \delta=8$ days, $R_{0}=\beta N / \gamma=$ 2.2. This model is used to find the number of ICU beds required in each country and predicting 
the death rates. Comparing their results for India with data. Their death predictions for the current time is very close to the real data for $R_{0}=0.8$. Wagh C. S. et al. [44] used SEIR with $\varepsilon=$ $0.2, y=0.1, \beta=0.21, y=0.1746, t=200, R_{0}=0$ and $I_{0}=1$. Predicting the epidemic peak could possibly reach the middle of July. Comparing their result with current data, the data varies with around $40 \%$ lower values than real data. Shaikh A. S. et al. [45] proposed a fractional order based SEIR with infected (symptomatic), people $\mathrm{I}(\mathrm{t})$, asymptotically infected $\mathrm{A}(\mathrm{t})$, and Quarantine $Q(t)$. They studied the dynamics of the novel coronavirus model suggested by Khan M. A. et al. [46]. The fractional-order model is as follows

$$
\begin{gathered}
{ }^{C F} D_{t}^{\tau} S(t)=\Delta-\lambda S-\alpha S(I+\beta A) / N-\gamma S Q \\
{ }^{C F} D_{t}^{\tau} E(t)=\alpha S(I+\beta A) / N+\gamma S Q-(1-\varphi) \delta E-\varphi \mu E-\lambda E \\
{ }^{C F} D_{t}^{\tau} I(t)=(1-\varphi) \delta E-(\sigma+\lambda) I \\
{ }^{C F} D_{t}^{\tau} A(t)=\varphi \mu E-(\rho+\lambda) A \\
{ }^{C F} D_{t}^{\tau} R(t)=\sigma I+\rho A-\lambda R \\
{ }^{C F} D_{t}^{\tau} Q(t)=\kappa I+\nu A-\eta Q
\end{gathered}
$$

Mandal M. et al. [47] used the SEIQR model on April 28. The parameters are selected as A $=1, \beta=1, \rho_{1}=-3.545, \rho_{2}=-7.5, \alpha=-0.138, \sigma=-0.144$. The proposed model is implemented in three major states Maharashtra, Delhi, and Tamil Nadu. But comparing the prediction results with real data, the predictions as of now are not following current trends and seem to be low by $30-50 \%$. Shah P. V. [48] used the SEIR model with $R_{0}=2.58, \beta=0.258$, $\varepsilon=0.2, \gamma=0.1, \mathrm{~N}=1339.2 \times 10^{6}, \mathrm{Q}=0.01$ to 0.1 . Comparing their prediction with real data, the current scenario might come under their results considering there are no interventions. 
Therefore, predicting the max values of 2,200,000 at around mid-August. Ravinder R. et al. [49] an adaptive interacting cluster-based SEIR (AICSEIR) model and applied it to multiple countries. The India model was constructed with $\mathrm{R}_{0}=2$ to 6 . According to the predictions of the model, the current pandemic size in India must be around 50 million but we have just crossed 100,000. Marimuthu Y. et al. [50] used the SEIR model on May 12. They used their previous model for TB patients in Delhi and added COVID-19 to it. Predicting COVID-19 infected TB cases. They predicted that due to the interventions the number can be lower by $30 \%$ and also delaying the peak cases by 44 days. Gupta [51] used SEIR-QDPA with Qa (detected and quarantined asymptomatic), Qs (detected and quarantined symptomatic), Ru (undetected recovered asymptomatic), $\mathrm{Ra}$ (recovered detected asymptomatic), Rs (recovered detected symptomatic), D (dead) and P (protected; nonsusceptible). They have used $\mathrm{R}_{0}=2.083$. Verma V. R. et al. [52] used a dynamic model based on SEIR for predicting the number of hospital beds required. Concluding that augmented testing of 500,000 tests per day during peak (mid-July) under a moderate lockdown scenario would lead to more reported cases $(5,500,000-6,000,000)$. Gupta R. et al. [53] proposed a model on April 3. They have used the SEIR and regression models to predict the number of cases with $R_{0}=2.01$. Comparing their prediction with real data none of the SEIR or regression models matches real data. There is a difference of about $40 \%$ in the regression model and about $50 \%$ in the SEIR model. Chatterjee K. et al. [54] used the following SEIQRD model for the outbreak of COVID-19

$$
\begin{gathered}
d S / d t=\beta I S \\
d E / d t=\beta I S-\varepsilon E
\end{gathered}
$$




$$
\begin{gathered}
d I / d t=\varepsilon E-\gamma I-d T-q I \\
d Q / d t=q I-q t Q-d Q \\
d R / d t=\gamma I+q T Q \\
d D / d t=d I+d Q
\end{gathered}
$$

Comparing the predictions with real data, the predictions seem to match with current data. Furthermore, the model predicts that the number will increase until July end and can reach up to 80 million.

\subsection{ARIMA and Exponential Models}

Rao A. S. et al. [55] constructed by Meyer wavelets. These wavelets are accompanied by the following function

$$
\psi(\omega)=\left\{\begin{array}{l}
\frac{1}{\sqrt{2 \pi}} \sin \left(\frac{\pi}{2} u\left(\frac{3|\omega|}{2 \pi}-1\right)\right) \mathrm{e}^{\frac{\mathrm{i} \omega}{2}} \text { if } 2 \pi / 3<|\omega|<4 \pi / 3 \\
\frac{1}{\sqrt{2 \pi}} \cos \left(\frac{\pi}{2} u\left(\frac{3|\omega|}{2 \pi}-1\right)\right) \mathrm{e}^{\frac{\mathrm{i} \omega}{2}} \text { if } 4 \pi / 3<|\omega|<8 \pi / 3 \\
0 \quad \text { otherwise }
\end{array}\right.
$$

Here $u(x)=0$ for $x<0$, and

$$
u(x)= \begin{cases}x & \text { for } x \in(0,1) \\ 1 & \text { for } x>1\end{cases}
$$

The model estimates that the number of COVID-19 infected would be 9225 (if there were 10 infected individuals as of 1 March 2020, who were not taking any precautions to spread), 17,986 (if there were $20 \%$ precautions) and 44,265 (if there were $50 \%$ precautions). Poonia N. et al. 
[56] proposed an Auto-Regressive Integrated Moving Average Model (ARIMA) Model and Holt's method for the prediction of infected people in India till May 1. On comparing with real data, the data is deviated by at least $20 \%$. Kumar P. et al. [57] proposed a model on April 2. They used ARIMA and Richard's model. They identified that by the end of April 2020, the incidence of new cases is predicted to be 5200 through the ARIMA model and 6378 through Richard model. But both the models predicted wrong as the real value reached around 30,000 by the end of April. Sharma, V. K. et al. [58] used ARIMA, exponential smoothing, and Holt-Winters models. Even though the model uses very recent data their predictions deviate from real data with $10 \%$. Mukhopadhyay S. et al. [59] proposed a model on May 3. According to their predictions, there must be 58277 cases by May 8, which in fact is very similar to recorded 59000 cases. Hence, this model might be useful in further predictions.

Chakraborty T. et al. [60] proposed a model that used a hybrid time series forecasting approach with wavelet-based techniques and ARIMA. The proposed hybrid model overcomes the deficiencies of the single time series models. They implemented the model in different countries. The predictions are relatively correct. Gupta R. et al. [61] proposed an ARIMA modeling and exponential smoothing methods have been implemented. Their predictions for the number of cases doesn't match with real data. There is a difference of more than $60 \%$. Shekhar H. [62] proposed a model on May 6. They used an exponential model for the prediction of daily infectious cases. The predicted value is not the same as real for whole India, but for states like Delhi, Gujrat, and Maharashtra it resembles real data very much. Rai B. et al. [63] and Dwivedi L. et al. [64] proposed an exponential model with Herd immunity. After calibrating the model, they predicted the active cases to reach 250,000 by April 30, But even the current data hasn't reached that number. Mishra P. et al. [65] proposed used an exponential regression model. Comparing the predictions with real data, the predictions matched real data for a long time then 
started to deviate. Currently, the model prediction lags within 10 days. Singh B. P. [66] proposed a model on May 5. They have used multiple growth models including linear growth, exponential growth and logistic growth models to predict future epidemic sizes. Comparing the prediction with real data, the predicted value comes at about $50 \%$ of the current data for all predicted values.

\subsection{Machine Learning based Models}

Sujatha R. [67] proposed a machine learning method based on feedforward artificial neural networks (FANN). The model is as follows

$$
\begin{gathered}
y=\varphi\left(\sum_{i=1}^{n} w_{i} x_{i}+b\right)=\varphi\left(w^{T} x+b\right) \\
Y_{t}=\alpha+\beta_{1} Y_{t-1}+\beta_{2} Y_{t-2}+\ldots+\beta_{p} Y_{t-p}+\varepsilon_{t}
\end{gathered}
$$

This model predicts 296072 confirmed cases, 4094 deaths, and 16136 recovered cases as on April 18, The model fails as real values are 15725,2546 , and 522, respectively according to real data.

Pasayat, A. K. et al. [68] proposed a model on May 20. They have applied machine learning algorithms to improve exponential growth and linear growth models. Compared with real data, the real data is following the exponential growth pattern given by the model. Tiwari $U$. et al. [69] proposed a machine learning-based model to predict the outcomes of COVID-19. The model's aim is to analyze whether the case of COVID-19 in India is going to be the same as in Italy or South Korea. The answer is yes. India might be going to face its worst days in the future if we look at the pattern of these countries and India too. Tiwari S. et al. [70] proposed a machine learning model. In the model, the outbreak in India has been predicted based on the 
pattern of China. Comparing the prediction with real data, the model predicts 68,978 cases by April 25, but the real values have reached around 24,000 . This may be due to the fact that people are more aware of the disease now and are following interventions, similar was introduced by Punn N. S. et al. [71].

Thakur A. et al. [72] proposed a model based on support vector machines and linear regression for predicting the expected cases. Comparing their predictions with real data, the real data matches with predictions with a difference of $10 \%$. Ogundokun R. O. et al. [5] proposed a model on May 26. The model uses ANNs in the estimation problem with multi-layer perceptions (MLPs), which uses the solitary tiers feed-forward network. This method is categorized by a system of 3 layers. The nodes in several tiers are as well identified as altering fundamentals. The outcome of the method is calculated employing the following mathematical expression

$$
y_{t}=\alpha_{0}+\sum_{j=1}^{q} \propto_{j} g\left(\beta_{0 j}+\sum_{i=1}^{p} \beta_{i j} y_{t-i}\right)+\varepsilon_{t, \forall t}
$$

They also incorporated other techniques like ARIMA, sigmoid function, and linear regression. Through the model, they calculated P-values for water and air. Tamang S. et al. [73] proposed an artificial neural network (ANN) model to predict the epidemic size of Different countries. For India, they have predicted a final size of 50,000 to 160,000 in India in 2 to 10 months. Currently, the numbers have crossed 140,000. Virk J. K. et al. [74] used lasso regression and logistic curve to predict the maximum cases and new cases per day. The predictions given by the model don't match real data. There is a difference of about $60 \%$. Hence, the model fails to predict the correct information. Tomar A. et al. [75] proposed a model on April 10. They have used data-driven estimation methods like long short-term memory (LSTM) and curve fitting for prediction of the number of COVID-19 cases in India 30 days ahead 
and the effect of preventive measures like social isolation and lockdown on the spread of COVID-19. The structure of LSTM consists of four gates i.e. input gate, forget gate, control gate, and output gate. Comparing the Predictions with real data, The predicted numbers are 80,000 on the 90th day but the numbers were half in real data. Furthermore, the result resembles real data with the transmission rate equal to 0.5 or lower. Bhardwaj R. [76] proposed a regression model to develop a data-driven extrapolation model. They used the proposed model for different countries. Comparing the result for India with real data, the model's prediction matches the real data with a difference of $10 \%$. Prabat D. [77] used the support vector regression model to predict the number of the total number of deaths, recovered cases, the cumulative number of confirmed cases, and the number of daily cases. Comparing the prediction with real data, the predictions don't match real data. There is a difference of $20-30 \%$ from real data.

Painuli D. et al. [78] proposed a rule-based model under a fuzzy inference system (FIS). This system uses 11 symptoms like age, sex, fever, dry cough, breathing problem, flu and cold, medical history, travel history, and two recently identified symptoms by some infected patients anosmia (lost sense of smell), loss of hearing ability. In this study, they shown that if fever is yes, cough is yes then the probability of coronavirus infection seems to be positive, similarly, if fever is greater than 38 and cough is dry which comes in the category of yes and patient is facing difficulty in breathing and he has also a travel history to some infected countries than the result shows the positive probability of coronavirus infection. Mahalle P. N. et al. [79] proposed a model on April 16. They proposed different predictive analytics techniques available for trend analysis, different models and algorithms, and their comparison. The prediction of COVID-19 using the Prophet algorithm indicates a faster spread in the short term. 


\subsection{Other Models}

There are many authors working on other than the above-mentioned models like arithmetic progression, polynomial based, network-based etc. Summary of these model is as follows:

Arti M. K. et al. [80] proposed a model on April 1. They have used the fact that transmissions are a chain process. Hence, They formulated a model based on arithmetic progression (AP). Some of their predicted values match real data including prediction about sudden surge from April 1 and the prediction for lockdown with an infectious rate equal to 0.6. Kumar D. et al. [81] used a similar approach proposed by Arti M. K. at. Al. [79]. They have used the model to study the data related to Madhya Pradesh, India, and tried to estimate the number of days in which the cases will be removed based on different recovery rates.

Ghosh S. [82] proposed a model on May 6. They have analyzed the effects of the pattern in the change factor, the first derivative of cases (i.e., the daily increase, for all of confirmed, deceased and recovered), as well as the second derivative (i.e. the increase in daily increases, for all of confirmed, deceased and recovered) for the raw data in the effectivity of predicting the number of total cases for the following day(s), for confirmed, recovered, as well as deceased cases. The results have a high accuracy of $83 \%$ until the date of April 30 . Also, the model is self-correcting as it improves its predictions every day. Roy S. et.al. [83] proposed a model on April 15. The present study is based on a differential equation that has been formed here to find how the number of asymptomatic patients increases with time. The number of symptomatic patients has been estimated from its solution. The predictions are correct till May 3 assuming they applied 40 days lockdown but the lockdown is extended. The model might be

correct. Ambikapathy B. et al. [84] proposed a model on May 7. The model consists of a 
framework of ordinary differential equations developed by incorporating the actual reported cases in 14 countries. After validation, the model was applied to predict COVID-19 transmission in India for different intervention scenarios in terms of lockdown for 4, 14, 21, 42, and 60 days. Their prediction 2-fold augmentation in the transmission is very similar to the real data.

Sivakumar A. [85] on May 2 and Menon V. K. et al. [86] on March 26 used the logistic model for predicting the epidemic size in India. The basic model of the logistic approach is as follows

$$
d C / d t=r C(1-c / K)
$$

where $C$ is accumulated the number of cases, $r$ is infection rate and $K$ is the final epidemic size. Sivakumar A. [85] used the model formulating factors themselves and adding certain constraints. Their prediction matches real data with a $5 \%$ difference. Hence, this model might be considered accurate. Mondal S. et al. [87] proposed a model on April 10. The model uses epidemic dynamics of COVID-19 through the sigmoid-type logistic function. Comparing the results with the real data, Most of the predictions are proven to be wrong. Gupta P. et al. [88] proposed a model based on Gaussian function

$$
y(t)=a e^{-\{(t-b) / c\}^{2}}
$$

This model is implemented in multiple countries. Comparing the results with real data, we can see that results are incorrect. Even in their conclusion itself, their predictions contradict on adding more data. Kumar A. [89] proposed a model on April 27. They proposed a Network-based system for displaying COVID-19 hazard at various topographical areas by utilizing movement and wind stream-based genuine information. They found that the movement towards local spots may bring about higher approaching cases in Uttar Pradesh and Bihar, 
while relocation to the export destinations can flood approaching cases in Karnataka, Maharashtra, and Haryana, and day by day streams are probably going to imperil the two arrangements of locales. Furthermore, it will assist with controlling the spread of COVID-19 by shutting explicit courses with a higher danger of pandemic spread. In any case, the model is presently not helpful as the government has halted all movement benefits in the nation.

Singh J. et al. [90] used the successive approximation method for the real-time data of India. Comparing their predicted cases with real data, the predictions match the real data. Patil N. L. [91] proposed a model on May 6. In this study, they group the data in a cycle of five and seven days. The method is useful where local transmission or community spread of disease is prevalent. The pattern of disease spread is delineated by the method based on reported data and doesn't consider the underlying unreported cases.

Roy S. [92] proposed a model on May 10. They have used an algebraic model to fit the current data and extrapolated it to predict future values. The predicted values are quite similar to the real data. Bhattacharyya A. et al. [93] proposed a model on April 25. They plotted daily new cases with the number of days spent after lockdown are fitted by linear and polynomial function and chose The best-fitted graph for interpretation and that is based on the parameters obtained from fitting. The forecast of maximum spreading possibility is discussed with linear and 4-degree polynomial fitting parameters. Compared to the data the model doesn't fit the real values and the predictions are not proposed in the model. Gupta S. [94] proposed a model on April 16. In the model, they collected the daily data of new cases in 50 US states between January 1-April 9, and also the corresponding weather information (i.e., temperature $(T)$ and absolute humidity $(\mathrm{AH})$ ). Distribution modeling of new cases across $\mathrm{AH}$ and $\mathrm{T}$ helped identify the narrow and vulnerable $\mathrm{AH}$ range. Assuming the US model to represent the world findings, we classified 
risky Indian states with expected $4<\mathrm{AH}<6 \mathrm{~g} / \mathrm{m} 3$ through all months of 2020 . Concluding their results can help predict vulnerable regions with high chances of weather-based spread in already affected countries, and countries with a high population, such as India, with the recent and rapidly rising spread.

Venkateswaran J. e. Al. [95] proposed a system dynamics (SD) model of the COVID-19 pandemic spread in India. Comparing the predictions with real data, the data matches with the $3 A$ policy. Furthermore, predicting that new cases per day might reach 25,000 per day until the first week of July.

\section{Discussion and Conclusions}

About 95 significant contributions have been reviewed to explore the outbreak of COVID-19 in India by the mathematical approach. The demonstration for a progressing episode of COVID-19 is as yet a difficult errand at this phase of the pandemic. We locate that most existing models of COVID-19 are ordinarily founded on plague dynamic models instead of the factual models or AI. In this study, various authors have included the lockdown as a part in their model $[8,20,24,37,52,74,83,84,93]$. They have shown that the susceptibility rate decreases due to the containment of people and providing help in maintaining social-distancing. Hence, we can conclude that the transmission rate has decreased due to lockdown implementation. The legislature carefully followed the initial two lockdowns. However, lockdown 3.0 - 4.0 the administration gave more unwinding to people in general and expanded the number of testing. Numerous forecasts before lockdown 3.0 are excellent. After lockdown 3.0, the charts for various states are extremely dissimilar. Also, many authors had shown the linkage of COVID-19 with environmental factors. The summary is as follows 
- The prediction of required ICU beds for COVID-19 patients was given [43, 52]. Being able to predict the number of ICU beds will help in the arrangement of proper facilities and providing better healthcare.

- The P-value of water and air for transmission is determined [5]. This study had shown that the P-value of water and air is enough that the transmit of medication through these mediums is possible and will reach the majority of the population.

- The study of temperature and humidity effect on COVID-19 spread in India was given [94]. They have shown that India's weather is unfavorable for people. Also, many states might have greater weather-based spread in the future.

- Also, most of the models have shown that there is a high death rate associated with COVID-19. Hence, the burying of these dead bodies may cause the soil-based transmission otherwise burning will cause various sorts of pollutants introduction in the environment.

There is as yet extensive vulnerability to evaluate the epidemiological attributes because of the novel idea of COVID-19 and the beginning period of the outbreak. Certain techniques present some inborn confinements because of deficient information and restricted information sources. Extra research is critically important to satisfy such research holes. We accept that as the pandemic arrives at the end and more information can be gathered, epidemiological models can be improved so as to introduce a genuine impression of the full picture. This survey will assume a significant job in future research to grow new scientific models for foreseeing and investigating the conduct of novel coronavirus (COVID-19).

The results of this review offer an overview of the current use of mathematical models in the context of the study of COVID-19 and indicate that direction of future research is needed. In the current work, we can without much of a stretch see which mathematical model has great 
outcomes in contrast with different models under Indian circumstances. The aftereffects of this survey offer an outline of the ebb and flow utilization of scientific models with regards to the investigation of COVID-19 and show that future research is required. In this investigation, we have attempted to sum up all the scientific models of COVID-19 in India which will help in giving an increasingly comprehensive structure to the analysts and specialists for picking up knowledge into the determinants of expectation and controlling the novel coronavirus. Furthermore, these models can be related to further studies depicting the effect of lockdown on environmental factors such as pollution index, and temperature variation due to a decrease in industrial and vehicle activities. Also, these models will help in expanding more information about the example and epidemiological example of the COVID-19 which can help the governing body in making certain steps concerning prosperity gauges like lockdown, purification, movement, medicinal administrations, and to be set up for the cutting-edge conditions. This review will help in scientific and information-driven demonstrated proof and bits of knowledge into the transmission, seriousness, and identity of the malady, which could promptly advise the dynamic in battling the sickness. 


\section{Reference}

1. MacKinnon, M., VanderKlippe, N., \& Robertson, G. (2020, April 25). Flattery and foot dragging: China's influence over the WHO under scrutiny. The Globe and Mail; The Globe and Mail. https://www.theglobeandmail.com/world/article-flattery-and-foot-dragging-chinas-influence-over-th e-who-under/

2. Shereen, M. A., Khan, S., Kazmi, A., Bashir, N., \& Siddique, R. (2020). COVID-19 infection: origin, transmission, and characteristics of human coronaviruses. Journal of Advanced Research. doi: https://doi.org/10.1016/j.jare.2020.03.005

3. Reuters Editorial. (2020, May 4). After retesting samples, French hospital discovers COVID-19 case from December. U.S.; Reuters. https://www.reuters.com/article/us-health-coronavirus-france/after-retesting-samples-french-hospi tal-discovers-covid-19-case-from-december-idUSKBN22G20L

4. Deslandes, A., Berti, V., Tandjaoui-Lambotte, Y., Alloui, C., Carbonnelle, E., Zahar, J. R., Brichler, S., \& Cohen, Y. (2020). SARS-CoV-2 was already spreading in France in late December 2019. International journal of antimicrobial agents, 106006. Advance online publication. https://doi.org/10.1016/j.ijantimicag.2020.106006

5. Fredericks, B. (2020, March 13). WHO says Europe is the new epicenter of coronavirus pandemic. New York Post; New York Post. https://nypost.com/2020/03/13/who-says-europe-is-new-epicenter-of-coronavirus-pandemicl

6. Ogundokun, R. O., \& Awotunde, J. B. (2020). Machine learning prediction for COVID-19 pandemic in India. doi: https://doi.org/10.1101/2020.05.20.20107847

7. MoHFW | Home. (2019). Retrieved May 24, 2020, from Mohfw.gov.in website: https://www.mohfw.gov.in

8. Singh, R., \& Adhikari, R. (2020). Age-structured impact of social distancing on the COVID-19 epidemic in India. ArXiv preprint arXiv:2003.12055.

9. Dhar, A. (2020). A critique of the Covid-19 analysis for India by Singh and Adhikari. arXiv preprint arXiv:2004.05373.

10. Ranjan, R. (2020). Predictions for COVID-19 outbreak in India using Epidemiological models. https://doi.org/10.1101/2020.04.02.20051466

11. Bhola, J., Venkateswaran, V. R., \& Koul, M. (2020). Corona Epidemic in the Indian context: Predictive Mathematical Modelling. medRxiv. doi: https://doi.org/10.1101/2020.04.03.20047175

12. Jakhar, M., Ahluwalia, P. K., \& Kumar, A. (2020). COVID-19 Epidemic Forecast in Different States of India using the SIR Model. medRxiv. doi: https://doi.org/10.1101/2020.05.14.20101725 
13. Malhotra, B., \& Kashyap, V. (2020). Progression of COVID-19 in Indian States-Forecasting Endpoints Using SIR and Logistic Growth Models. medRxiv. doi: https://doi.org/10.1101/2020.05.15.20103028

14. fitVirusCOVID19 - File Exchange - MATLAB Central. (2020, May 12). Retrieved May 20, 2020, from Mathworks.com website: https://www.mathworks.com/matlabcentral/fileexchange/74658-fitviruscovid19

15. Batista, M. (2020). Estimation of the final size of the COVID-19 epidemic. MedRxiv. doi, 10, 16-20023606. doi: https://doi.org/10.1101/2020.02.16.20023606

16. Tiwari, A. (2020). Modeling and analysis of the COVID-19 epidemic in India. medRxiv. doi: https://doi.org/10.1101/2020.04.12.20062794

17. Yadav, R. S. (2020). Mathematical Modeling and Simulation of SIR Model for COVID- 2019 Epidemic Outbreak: A Case Study of India. medRxiv. doi: https://doi.org/10.1101/2020.05.15.20103077

18. KUMAR, S. (2020). Prediction of Pandemic COVID-19 situation in Maharashtra, India. medRxiv. doi: https://doi.org/10.1101/2020.04.10.20056697

19. SINGH, A., Barai, A. K., \& Shinde, A. (2020). Modeling and data-based analysis of COVID-19 outbreak in India: a study on the impact of social distancing measures. medRxiv. doi: https://doi.org/10.1101/2020.05.12.20099184

20. Kumar, K., Meitei, W. B., \& Singh, A. (2020). Projecting the future trajectory of COVID-19 infections in India using the susceptible-infected-recovered (SIR) model. https://www.iipsindia.ac.in/sites/default/files/iips covid19 pfti.pdf

21. Maji, C. (2020). Modes of transmission of COVID-19 outbreak- a mathematical study. https://doi.org/10.1101/2020.05.16.20104315

22. Pujari, B. S., \& Shekatkar, S. M. (2020). Multi-city modeling of epidemics using spatial networks: Application to 2019-nCov (COVID-19) coronavirus in India. https://doi.org/10.1101/2020.03.13.20035386

23. Mandal, M., \& Mandal, S. (2020). COVID-19 pandemic scenario in India compared to China and the rest of the world: data-driven and model analysis. https://doi.org/10.1101/2020.04.20.20072744

24. Dhanwant, J. N., \& Ramanathan, V. (2020). Quantitative Estimation of Disruption in Social Contact Structure and its Effect in COVID-19 Spread in India. https://doi.org/10.1101/2020.04.27.20081620

25. Das, S. (2020). Prediction of COVID-19 disease progression in India: Under the effect of national lockdown. arXiv preprint arXiv:2004.03147.

26. Deo, V., Chetiya, A. R., Deka, B., \& Grover, G. (2020). Forecasting Transmission Dynamics of COVID-19 Epidemic in India under Various Containment Measures- A Time-Dependent State-Space SIR Approach. https://doi.org/10.1101/2020.05.08.20095877 
27. Ghosh, P., Ghosh, R., \& Chakraborty, B. (2020). COVID-19 in India: State-wise Analysis and Prediction. MedRxiv. doi: https://doi.org/10.1101/2020.04.24.20077792

28. Rajesh, A., Pai, H., Roy, V., Samanta, S., \& Ghosh, S. (2020). CoVID-19 prediction for India from the existing data and SIR (D) model study. medRxiv. doi: https://doi.org/10.1101/2020.05.05.20085902

29. PRASAD, J. (2020). A data-first approach to modeling Covid-19. https://doi.org/10.1101/2020.05.22.20110171

30. Lathika Rajendrakumar, A., Thakarakkattil Narayanan Nair, A., Nangia, C., Kumar Chourasia, P., Kumar Chourasia, M., Ghouse Syed, M., Sasidharan Nair, A., B Nair, A., \& Fazaludeen Koya, M. S. (2020). Epidemic Landscape and Forecasting of SARS-CoV-2 in India. https://doi.org/10.1101/2020.04.14.20065151

31. Chatterjee, S., Sarkar, A., Chatterjee, S., Karmakar, M., \& Paul, R. (2020). Studying the progress of the COVID-19 outbreak in India using the SIRD model. https://doi.org/10.1101/2020.05.11.20098681

32. Ranjan, R. (2020). Estimating the Final Epidemic Size for COVID-19 Outbreak using Improved Epidemiological Models. MedRxiv. doi: https://doi.org/10.1101/2020.04.12.20061002

33. Maji, A., Sushma, M. B., \& Choudhari, T. (2020). The implication of the Inter-State Movement of Migrant Workers during COVID 19 Lockdown using Modified SEIR Model. arXiv preprint arXiv:2005.04424.

34. Sardar, T., Nadim, S. S., \& Chattopadhyay, J. (2020). Assessment of 21 days lockdown effect in some states and overall India: a predictive mathematical study on COVID-19 outbreak. ArXiv preprint arXiv:2004.03487.

35. Senapati, A., Rana, S., Das, T., \& Chattopadhyay, J. (2020). Impact of intervention on the spread of COVID-19 in India: A model-based study. ArXiv preprint arXiv:2004.04950.

36. Pal, D., Ghosh, D., Santra, P. K., \& Mahapatra, G. S. (2020). Mathematical Analysis of a COVID-19 Epidemic Model by using Data-Driven Epidemiological Parameters of Diseases Spread in India. medRxiv. doi: https://doi.org/10.1101/2020.04.25.20079111

37. Menon, A., Rajendran, N. K., Chandrachud, A., \& Setlur, G. (2020). Modeling and simulation of COVID-19 propagation in a large population with specific reference to India. medRxiv. doi: https://doi.org/10.1101/2020.04.30.20086306

38. Singh, A., Dey, J., \& Bhardwaj, S. (2020). Is this the beginning or the end of the COVID-19 outbreak in India? A data-driven mathematical model-based analysis. medRxiv. doi: https://doi.org/10.1101/2020.04.27.20081422

39. Sinha, D. N. (2020). Mathematical Modeling to Estimate the Reproductive Number and the Outbreak Size of COVID-19: The case of India and the World. DOI: 10.21203/rs.3.rs-26261/v1

40. Khatua, D., De, A., Kar, S., Samanta, E., \& Mandal, S. M. (2020). A Dynamic Optimal Control Model for SARS-CoV-2 in India. Available at SSRN 3597498. 
41. Mandal, S., Bhatnagar, T., Arinaminpathy, N., Agarwal, A., Chowdhury, A., Murhekar, M., Gangakhedkar, R. R., \& Sarkar, S. (2020). Prudent public health intervention strategies to control the coronavirus disease 2019 transmission in India: A mathematical model-based approach. The Indian journal of medical research, 151(2 \& 3), 190-199. https://doi.org/10.4103/ijmr. IJMR 50420

42. Pai, C., Bhaskar, A., \& Rawoot, V. (2020). Investigating the dynamics of COVID-19 pandemic in India under lockdown. arXiv preprint arXiv:2004.13337.

43. Chowdhury, R., Heng, K., Shawon, M. S. R., Goh, G., Okonofua, D., Ochoa-Rosales, C., Gonzalez-Jaramillo, V., Bhuiya, A., Reidpath, D., Prathapan, S., Shahzad, S., Althaus, C. L., Gonzalez-Jaramillo, N., \& Franco, O. H. (2020). Dynamic interventions to control COVID-19 pandemic: a multivariate prediction modelling study comparing 16 worldwide countries. European Journal of Epidemiology, 35(5), 389-399. https://doi.org/10.1007/s10654-020-00649-w

44. Wagh, C.S.; Mahalle, P.N.; Wagh, S.J. Epidemic Peak for COVID-19 in India, 2020. Preprints 2020, 2020050176. doi: 10.20944/preprints202005.0176.v1

45. Shaikh, A.S.; Shaikh, I.N.; Nisar, K.S. A Mathematical Model of COVID-19 Using Fractional Derivative: Outbreak in India with Dynamics of Transmission and Control. Preprints 2020, 2020040140. doi: 10.20944/preprints202004.0140.v1

46. Khan, M. A., \& Atangana, A. (2020). Modeling the dynamics of novel coronavirus (2019-nCov) with fractional derivatives. Alexandria Engineering Journal. doi: https://doi.org/10.1016/j.aej.2020.02.033

47. Mandal, M., Jana, S., Nandi, S. K., Khatua, A., Adak, S., \& Kar, T. K. (2020). A model-based study on the dynamics of COVID-19: Prediction and control. Chaos, Solitons \& Fractals, 109889.

48. Shah, P. V. (2020). Prediction of the Peak, Effect of Intervention, and Total Infected by the coronavirus Disease in India. medRxiv. doi: https://doi.org/10.1101/2020.04.20.20072793

49. Ravinder, R., Singh, S., Bishnoi, S., Jan, A., Sinha, A., Sharma, A., Kodamana, H., \& Krishnan, N. M. A. (2020). An Adaptive, Interacting, Cluster-Based Model Accurately Predicts the Transmission Dynamics of COVID-19. https://doi.org/10.1101/2020.04.21.20074211

50. Marimuthu, Y., Nagappa, B., Sharma, N., Basu, S., \& Chopra, K. K. (2020). COVID-19 and Tuberculosis: A mathematical model based forecasting in Delhi, India. Indian Journal of Tuberculosis. doi: 10.1016/j.ijtb.2020.05.006

51. Gupta, M., Mohanta, S. S., Rao, A., Parameswaran, G. G., Agarwal, M., Arora, M., Mazumder, A., Lohiya, A., Behera, P., Bansal, A., Kumar, R., Meena, V. P., Tiwari, P., Mohan, A., \& Bhatnagar, S. (2020). Transmission dynamics of the COVID-19 epidemic in India and modelling optimal lockdown exit strategies. https://doi.org/10.1101/2020.05.13.20096826

52. Verma, V. R., Saini, A., Gandhi, S., Dash, U., \& Koya, D. M. S. F. (2020). Projecting Demand-Supply Gap of Hospital Capacity in India in the face of COVID-19 pandemic using the Age-Structured Deterministic SEIR model. https://doi.org/10.1101/2020.05.14.20100537 
53. Gupta, R., Pandey, G., Chaudhary, P., \& Pal, S. K. (2020). SEIR and Regression Model-based COVID-19 outbreak predictions in India. https://doi.org/10.1101/2020.04.01.20049825

54. Chatterjee, K., Chatterjee, K., Kumar, A., \& Shankar, S. (2020). Healthcare impact of COVID-19 epidemic in India: A stochastic mathematical model. Medical Journal Armed Forces India, 76(2), 147-155. https://doi.org/10.1016/j.mjafi.2020.03.022

55. Rao, A. S., Krantz, S. G., Kurien, T., Bhat, R., \& Kurapati, S. (2020). Model-based retrospective estimates for COVID-19 or coronavirus in India: continued efforts required to contain the virus Spread. Current Science, 118(7), 1023-1025.

56. Poonia, N., \& Azad, S. (2020). Short-term forecasts of COVID-19 spread across Indian states until 1 May 2020. arXiv preprint arXiv:2004.13538.

57. Kumar, P., Singh, R. K., Nanda, C., Kalita, H., Patairiya, S., Sharma, Y. D., Rani, M., \& Bhagavathula, A. S. (2020). Forecasting COVID-19 impact in India using pandemic waves Nonlinear Growth Models. https://doi.org/10.1101/2020.03.30.20047803

58. Sharma, V. K., \& Nigam, U. (2020). Modeling of Covid-19 cases in India using Regression and Time Series models. doi: https://doi.org/10.1101/2020.05.20.20107540.

59. Mukhopadhyay, S., \& Chakraborty, D. (2020). Estimation of undetected COVID-19 infections in India. medRxiv. doi: https://doi.org/10.1101/2020.04.20.20072892

60. Chakraborty, T., \& Ghosh, I. (2020). Real-time forecasts and risk assessment of novel coronavirus (COVID-19) cases: A data-driven analysis. Chaos, Solitons \& Fractals, 109850. DOI: https://doi.org/10.1016/j.chaos.2020.109850

61. Gupta, R., \& Pal, S. K. (2020). Trend Analysis and Forecasting of COVID-19 outbreak in India. https://doi.org/10.1101/2020.03.26.20044511

62. Shekhar, H. (2020). Prediction of Spreads of COVID-19 in India from Current Trend. medRxiv. doi: https://doi.org/10.1101/2020.05.01.20087460

63. Rai, B., Shukla, A., \& Dwivedi, L. K. (2020). COVID-19 in India: Predictions, Reproduction Number, and Public Health Preparedness. medRxiv. doi: https://doi.org/10.1101/2020.04.09.20059261

64. Dwivedi, L. K., Rai, B., \& Shukla, A. (2020, February 2). Assessing the Impact of Complete Lockdown on COVID-19 Infections in India and its Burden on Public Health Facilities. Working Papers; eSocialSciences. https://ideas.repec.org/p/ess/wpaper/id13051.html

65. Mishra, P. K., \& Mishra, S. (2020). A deductive approach to modeling the spread of COVID-19. https://doi.org/10.1101/2020.03.26.20044651

66. Singh, B. P. (2020). Forecasting Novel Corona Positive Cases in India using Truncated Information: A Mathematical Approach. https://doi.org/10.1101/2020.04.29.20085175

67. Sujatha, R., \& Chatterjee, J. (2020). A machine learning methodology for forecasting of the COVID-19 cases in India. doi: https://doi.org/10.36227/techrxiv.12143685.v1 
68. Pasayat, A. K., Pati, S. N., \& Maharana, A. (2020). Predicting the COVID-19 positive cases in India with concern to Lockdown by using Mathematical and Machine Learning based Models. https://doi.org/10.1101/2020.05.16.20104133

69. Tiwari, Upendra \& Khan, Rizwan. (2020). Role of Machine Learning to Predict the Outbreak of Covid-19 in India. Xi'an Jianzhu Keji Daxue Xuebao/Journal of Xi'an University of Architecture \& Technology. 12. 2663-2669.

70. Tiwari, S., Kumar, S., \& Guleria, K. (2020). Outbreak trends of coronavirus (COVID-19) in India: A Prediction. Disaster medicine and public health preparedness, 1-9. DOI: 10.1017/dmp.2020.115

71. Punn, N. S., Sonbhadra, S. K., \& Agarwal, S. (2020). COVID-19 Epidemic Analysis using Machine Learning and Deep Learning Algorithms. https://doi.org/10.1101/2020.04.08.20057679

72. Thakur A, Singh R, Gehlot A, Akram SV, Das PK. Comprehensive Data Analysis and Forecasting of Covid19 Outbreak in India. JMIR Preprints. 27/04/2020:19680. DOI: 10.2196/preprints. 19680

73. Tamang, S. ., Singh, P. ., \& Datta, B. (2020). Forecasting of Covid-19 cases based on prediction using artificial neural network curve fitting technique. Global Journal of Environmental Science and Management, 6(Special Issue (Covid-19)), 53-64. https://doi.org/10.22034/GJESM.2019.06.SI.06

74. Singh Virk, J., Ali, S. A., \& Kaur, G. (2020). Recent update on COVID-19 in India: Is locking down the country enough? https://doi.org/10.1101/2020.04.06.20053124

75. Tomar, A., \& Gupta, N. (2020). Prediction for the spread of COVID-19 in India and the effectiveness of preventive measures. Science of The Total Environment, 138762. https://doi.org/10.1016/j.scitotenv.2020.138762

76. Bhardwaj, R. (2020). A Predictive Model for the Evolution of COVID-19. https://doi.org/10.1101/2020.04.13.20063271

77. Parbat, D., \& Chakraborty, M. (2020). A Python Based Support Vector Regression Model for Prediction of COVID19 Cases in India. Available at SSRN 3591840.

78. Painuli, D., Mishra, D., Bhardwaj, S., \& Aggarwal, M. Fuzzy Rule-Based System to predict COVID19-A Deadly Virus. way, 3(4), 5. ISSN: 2394-0913

79. Mahalle, P. N., Sable, N. P., Mahalle, N. P., \& Shinde, G. R. (2020). Predictive Analytics of COVID-19 Using Information, Communication, and Technologies. https://doi.org/10.20944/preprints202004.0257.v1

80. Arti, M. K., \& Bhatnagar, K. Modeling and Predictions for COVID 19 Spread in India. ResearchGate, DOI: 10.13140/RG.2.2.11427.81444

81. Kumar, D., Kumari, P., \& Sarangi, S. Mathematical modeling of COVID-19 spread and stabilization in Madhya Pradesh, India: A fundamental approach. DOI: 10.13140/RG.2.2.31266.38089 
82. Ghosh, S. (2020). Predictive Model with Analysis of the Initial Spread of COVID-19 in India. medRxiv. doi: https://doi.org/10.1101/2020.05.02.20088997

83. Roy, S., \& Roy Bhattacharya, K. (2020). The spread of COVID-19 in India: A Mathematical Model. Available at SSRN 3587212. DOI: https://doi.org/10.46243/jst.2020.v5.i3.pp41-47

84. Ambikapathy, B., \& Krishnamurthy, K. (2020). Mathematical Modelling to Assess the Impact of Lockdown on COVID-19 Transmission in India: Model Development and Validation. JMIR Public Health and Surveillance, 6(2), e19368. doi: 10.2196/19368

85. Sivakumar, A. Review of mathematical models to predict the rate of spread and control of COVID 19 in India. DOI: 10.13140/RG.2.2.23670.75844

86. Menon, V. K. (2020). Prediction of the number of cases expected and estimation of the final size of the coronavirus epidemic in India using the logistic model and genetic algorithm. arXiv preprint arXiv:2003.12017.

87. Mondal, S., \& Ghosh, S. (2020). Possibilities of exponential or Sigmoid growth of Covid19 data in different states of India. medRxiv. DOI: 10.13140/RG. 2.2.11397.50406

88. Gupta, P., Sharma, K. K., Joshi, S. D., \& Goyal, S. (2020). A data-driven method to detect the flattening of the COVID-19 pandemic curve and estimating its ending life-cycle using only the time-series of new cases per day. medRxiv. doi: https://doi.org/10.1101/2020.05.15.20103374

89. Kumar, A. (2020). Modeling the geographical spread of COVID-19 in India using a network-based approach. medRxiv. doi: https://doi.org/10.1101/2020.04.23.20076489

90. Singh, J., Ahluwalia, P. K., \& Kumar, A. (2020). Mathematical Model Based COVID-19 Prediction in India and its Different States. medRxiv. doi: https://doi.org/10.1101/2020.05.16.2010423

91. Patil, N. L., \& Gangavati, L. R. (2020). Evaluating growth patterns and assessing future scenarios of the COVID-19 epidemic of India. https://doi.org/10.1101/2020.05.02.20087544

92. Roy, S. (2020). The spread of COVID-19 in India: A Simple Algebraic Study. https://doi.org/10.1101/2020.05.10.20097691

93. Bhattacharyya, A., Bhowmik, D., \& Mukherjee, J. (2020). Forecast and interpretation of daily affected people during 21 days lockdown due to COVID 19 pandemic in India. https://doi.org/10.1101/2020.04.22.20075572

94. Gupta, S., Raghuwanshi, G. S., \& Chanda, A. (2020). Effect of weather on COVID-19 spread in the US: A prediction model for India in 2020. Science of The Total Environment, 728, 138860. https://doi.org/10.1016/j.scitotenv.2020.138860

95. Venkateswaran, J., \& Damani, O. (2020). Effectiveness of Testing, Tracing, Social Distancing, and Hygiene in Tackling Covid-19 in India: A System Dynamics Model. arXiv preprint arXiv:2004.08859. 